\title{
Physiological Noise in Near-infrared Spectroscopy: Implications for Optical Brain Computer Interfacing
}

\author{
S. Coyle ${ }^{1}$, T. Ward ${ }^{1}$, C. Markham ${ }^{2}$ \\ ${ }^{1}$ Department of Electronic Engineering, National University of Ireland, Maynooth, Ireland \\ ${ }^{2}$ Department of Computer Science, National University of Ireland, Maynooth, Ireland
}

\begin{abstract}
Near-infrared spectroscopy is a non-invasive optical method used to detect functional activation of the cerebral cortex. Cognitive, visual, auditory and motor tasks are among the functions that have been investigated by this technique in the context of optical brain computer interfacing. In order to determine whether the optical response is due to a stimulus, it is essential to identify and reduce the effects of physiological noise. This paper characterizes noise typically present in optical responses and reports signal processing approaches used to overcome such noise.
\end{abstract}

Keywords-near-infrared spectroscopy, cerebral haemodynamics, physiological noise, brain computer interface

\section{INTRODUCTION}

Brain-computer interfaces (BCIs) are essential in improving the lifestyles of those suffering from neuromuscular impairments. They allow such users to communicate through thought processes alone. Neural activity within the motor cortex is a typical physiological signal used in this context and we have used non-invasive optical techniques as a safe and practical system to record direct measures of such signals in humans. A full description of a BCI we have developed using the above principles, is given elsewhere [1]. Our system uses near-infrared sensing techniques to detect characteristic haemodynamic responses in the motor cortex due to motor imagery and translates such responses to control an external device. The current system is easy to use but slow (3 bits/minute) and has a symbol error rate of 0.20 . This error rate is primarily due to misclassification of physiological noise signals as false 'positives'. An extension of the current system to multiple sites across the head will no doubt improve this performance as differential signals can allow localised changes of interest to be more easily seen. However from a single site an improvement in performance can certainly be achieved through improved suppression of the physiological noise present during optical sensing. In order to do this a signal processing noise model is required. In this paper such a model is tentatively proposed with some application to data acquired during BCI experiments. In addition, taxonomy of physiological noise in such optical interfacing is explored.

Near-infrared light can penetrate the human adult head to sufficient depths so as to allow functional mapping of the cerebral cortex. Cortical activity leads to haemodynamic changes associated with an increase in cerebral metabolism
[2]. These changes in tissue oxygenation associated with brain activity modulate the absorption and scattering of the NIR photons. Oxy-haemoglobin and deoxy-haemoglobin, which are the main absorbers, have different attenuation spectra. This means that multi-wavelength systems can monitor changes in such chromophore concentrations [3] giving qualitative measures of brain activity. This is the basis of the near-infrared techniques described in this communication.

Haemodynamic responses resulting from cerebrovascular activity cause changes in transmitted light intensity of approximately $1 \%$. The optical responses are observed as negative peaks in detected light intensity after 5-8 seconds following the onset of a stimulus e.g. response of motor cortex due to hand movement [4]. Recent studies have reported a fast optical response that is thought to be due to scattering changes related to neuronal firings, and induce signal changes of $0.01-0.1 \%$ [5]. Such intrinsic optical signals are of the order of milliseconds, are highly localized and require considerable averaging at present to achieve acceptable signal to noise ratios.

In comparison to electrical or magnetic technologies, it is quite easy to reduce the effects of environmental noise using optical techniques. One of the great benefits of optical methods is the lack of mains-induced electrical interference - the dreaded main "hum". Physiological effects tend to be the greatest sources of noise, namely cardiac pulses, respiratory effects and cerebral vasomotion, a spontaneous low frequency oscillation. A good description of the constituent oscillations in cerebral haemodynamics is given in [6] where the characteristics of physiological noise have been investigated using near-infrared techniques.

In the next section a description of the experimental setup is given. Section III describes the signals obtained, characterizes the different physiological noise sources present before describing signal-processing technique we have used to suppress them. Section IV presents a discussion of the results and their implications for optical BCIs.

\section{Methodology}

1) NIR measurement system: Our current NIR system consists of a single channel arrangement operating at two different wavelengths. The light sources, LEDs at $700 \mathrm{~nm}$ and $880 \mathrm{~nm}$, are placed in direct contact with the scalp. The LEDs are modulated at $4 \mathrm{kHz}$ and $5 \mathrm{kHz}$ and emit a combined average power of less than $2 \mathrm{~mW} / \mathrm{mm}^{2}$. 
Modulation of the light enables lock-in detection to be performed at each wavelength. The detector is an avalanche photodiode (APD) (Hamamatsu C5460-01) that is connected $3-4 \mathrm{~cm}$ from the light source via a $3 \mathrm{~mm}$ diameter fibre optic bundle. Two lock-in amplifiers (Ametek 5210) are used to obtain the intensities of the two wavelengths of light. The data is recorded at a sampling rate of $100 \mathrm{~Hz}$ by a $16-b i t \mathrm{~A} / \mathrm{D}$ data acquisition card (Keithley PCMCIA16AI).

2) Minimizing system and environmental noise: The LEDs are biased and modulated so as to produce a sine wave signal from the detector. Square wave modulation of the LEDs adds harmonic noise to the signal and runs the risk of cross talk between channels e.g. modulation of an LED at $1 \mathrm{kHz}$ with a square wave produces a sizeable noise signal at $3 \mathrm{kHz}$ and $5 \mathrm{kHz}$. While modulation of the light enables lockin detection, it also reduces noise due to ambient light, which can originate from daylight (DC), room lights $(100 \mathrm{~Hz})$ and computer monitors $(60 / 72 \mathrm{~Hz})$. The subject should also be shielded from such noise sources in so far as possible, and it is essential to prevent stray light from the source reaching the detector. Motion artifacts can be a problem as slight movements of the optodes on the scalp can cause large changes in the optical signal, due to variations in optical path. It is therefore important to ensure robust coupling of optodes to the subject's head.

3) Experimental Protocol: Cortical activation due to motor function was used in this study. Following an initial rest period to establish baseline conditions, subjects were asked to perform either actual or imagined hand movement tasks for a period of 20s. A 20s rest interval was allowed between each task. The optical response was measured at position $\mathrm{C} 3$ or $\mathrm{C} 4$ (areas associated with the right and left hand respectively) above the cerebral hemisphere contralateral to hand movement. A full account of this protocol is described in [4].

\section{RESULtS}

\section{A. Spectral characteristics of the optical response}

Analysis of the spectrum of optical signals helps to identify the quasi-periodic noise sources present. Spectral analysis of the optical signal acquired from a single subject is shown in Fig. 1. Cardiac signals are typically centered around $0.5-2 \mathrm{~Hz}$ ( $\mathrm{P} 3$ in Fig. 1), respiration between $0.2-0.4 \mathrm{~Hz}$ (P2 in Fig.1) and the Mayer wave around $0.1 \mathrm{~Hz}(\mathrm{P} 1$ in Fig.1). The results are consistent with the study reported in [6].

Following identification of the noise sources, we developed algorithms in an attempt to minimize the effects of such noise. This is discussed in the Sections B and C.

\section{B. Cardiac Pulse Removal}

Photoplethysmography using near-infrared light has been used for some time to measure the rate of blood vessel
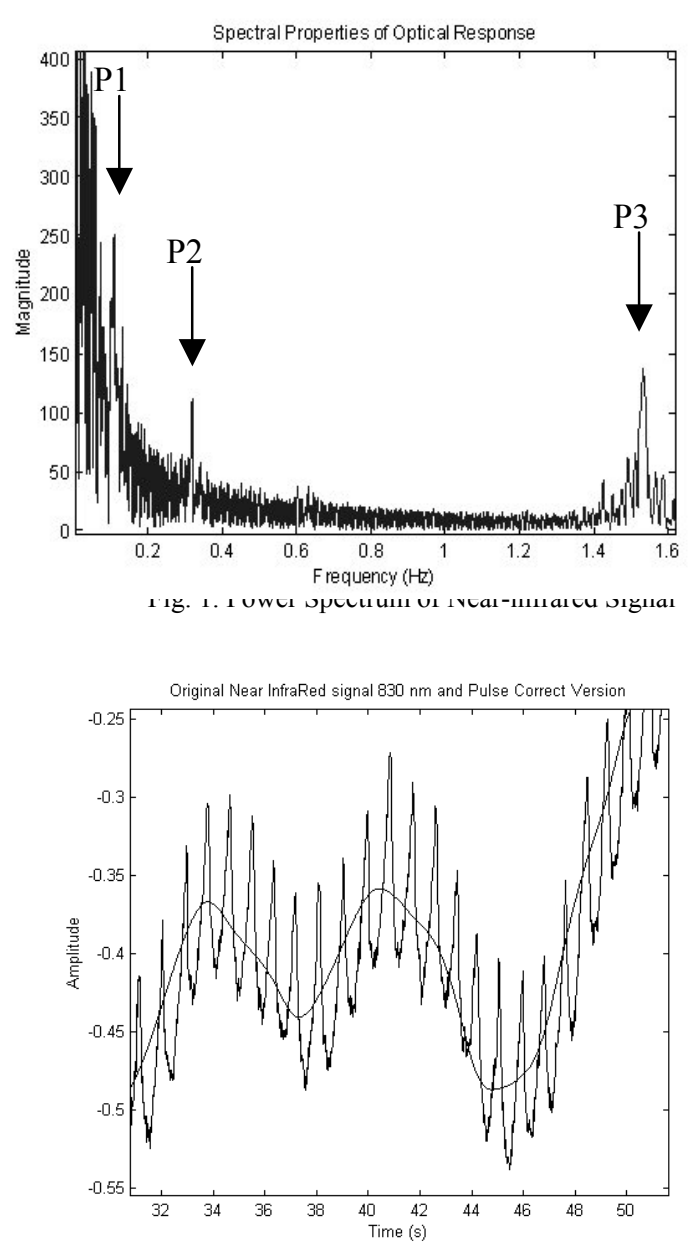

Fig. 2. Original Near-infrared signal and pulse corrected signal

pulsations, particularly in a clinical environment [8]. However, from a functional imaging perspective the cardiac pulse is considered to be a source of noise, particularly when looking for fast intrinsic signals [9].

The cardiac peaks are identified, to yield a set of heartbeat intervals. Under the assumption that the haemodynamic response is slowly and smoothly varying relative to the cardiac pulse signal, we then compute the mean of the average signal during each heartbeat interval. To obtain the smooth pulse corrected signal we interpolate using a cubic spline against the original time vector. This algorithm is described in detail elsewhere [10].

To highlight the benefits of the cardiac pulse removal algorithm over using a standard low pass filter, Fig. 6 shows the spectra of a section of the original data, the low pass filtered data, and the mean removed and spline-connected data. For this purpose, the chirp z-transform [11] was employed, as it is a most useful instrument for examining the spectral properties of our optical signals where the frequencies involved are quite low and closely spaced. 

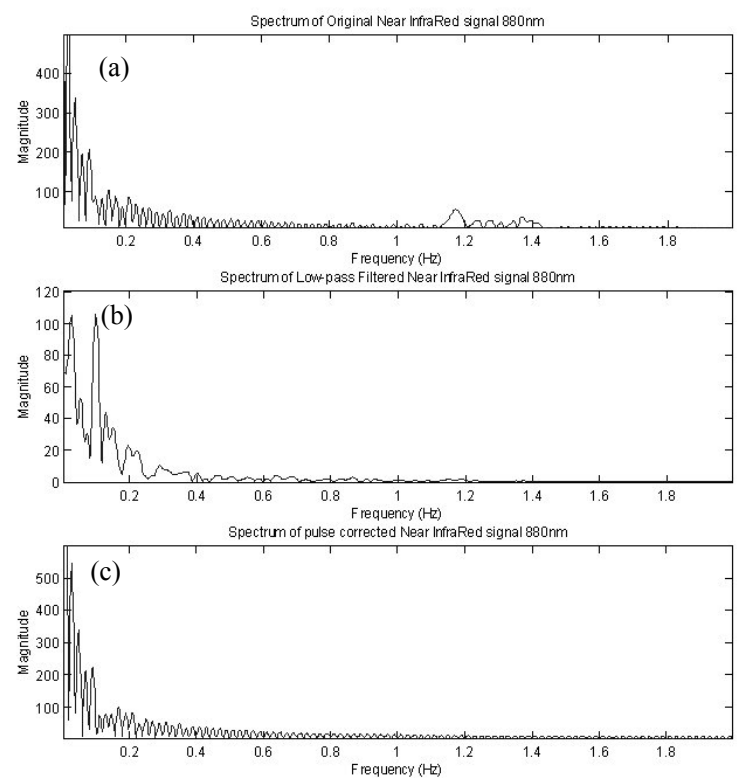

Fig. 4 Spectrum of (a) original near-infrared signal, (b) low-pass filtered signal, (c) pulse corrected signal

\section{Mayer wave}

Spontaneous low frequency oscillations around $0.1 \mathrm{~Hz}$ have been termed the Mayer wave [6]. These oscillations, also referred to as vasomotion, V-signal and spontaneous oscillations, been reported by various studies using different techniques $[6,7,12]$. Reference [12] tabulates a summary of studies using different physiological measurement systems in different species. The origin of this signal is not clearly understood at present. It was thought to represent baroreceptor mediated blood pressure control and later interpreted to be originating from a central oscillator [13]. The magnitude of these oscillations renders them a significant noise source in functional activation studies [6], and particularly for optical brain computer interfacing where single-trial events are necessary to ensure optimum speed.

In an effort to reduce the influence of the low oscillations on the optical data, we have developed a signal processing noise model. The signal processing relies on an IEEE Standard 1057 algorithm for fitting the parameters of a sine wave to noisy data [14]. Our model assumes that the Mayer wave $\mathrm{m}(\mathrm{t})$, being quasi-periodic in nature, can be represented as a sine wave

$$
\mathrm{m}(\mathrm{t})=\alpha \sin (\omega \mathrm{t}+\phi) .
$$

$\alpha$ is the amplitude, $\omega$ is the frequency of the oscillation and $\phi$ is its phase relative to time $t=0$. Based on data acquired from various subjects, this appears to be a reasonable assumption for small time intervals (10-20s).

The assumption that $\alpha$ and $\omega$ are constant during the analysis windows has been shown to be true providing there are no gross changes in the subject's position such as moving from supine to seated [15].
The optical data containing the sequence $\mathrm{y}_{0}, \ldots, \mathrm{y}_{\mathrm{N}}$ taken at times $\mathrm{t}_{0}, \ldots, \mathrm{t}_{\mathrm{N}}$ can be modeled by

$$
\mathrm{y}_{\mathrm{k}}(\mathrm{A}, \mathrm{B}, \mathrm{C}, \omega)=\mathrm{A} \cos \omega \mathrm{t}_{\mathrm{k}}+\mathrm{Bsin} \mathrm{t}_{\mathrm{k}}+\mathrm{C}
$$

where $\mathrm{A}=\alpha \sin \phi$ and $\mathrm{B}=\alpha \cos \phi$ are unknown constants, $\omega$ is the angular frequency of the oscillation and $\mathrm{C}$ is the residual signal.

For a given segment of data, the four unknown parameters can be found using a least squares fit algorithm $[15,16]$, taking the frequency to be $0.1 \mathrm{~Hz}$ as an initial guess for $\omega$. In order to take into account the time-varying frequency and amplitude of the oscillations, a moving window of ten second duration is applied; yielding the best fit values of $\mathrm{A}, \mathrm{B}, \mathrm{C}$ and $\omega$ for each second in time. Applying this algorithm to single trials involving motor function can significantly reduce the effects of the Mayer wave, as shown in Fig. 5. Data segments where no stimulus (hand movement) was present, but where the amplitude of the Mayer wave could be interpreted as an event occurrence are shown in Fig. 6(a) and Fig. 6(b). As our optical BCI currently relies on a straightforward thresholding approach for event classification, applying this algorithm can reduce the bit error rate.

\section{DISCUSSION}

Near-infrared techniques, being of a non-invasive nature, are ideal for continuous physiological monitoring and hence optical brain computer interface applications [1]. The current system we have developed is very accessible but slow (3 bits/minute) and has a symbol error rate of 0.20 . Given the time-course of the cerebral haemodynamic response $(5-8 \mathrm{sec})$, the speed at which such a BCI can operate is limited to an extent. In order to achieve optimum speed, single trial events must be used for BCI control. In order to do, and also to avoid sacrificing the accuracy of the system, it is essential to improve the signal to noise ratio. This is done by minimizing system noise and environmental noise, as discussed in Section II, and also by developing signal processing techniques to reduce physiological noise effects, some possible methods of achieving this are presented in Section III. Although the algorithm presented in subsection B is used to remove the Mayer wave $(0.1 \mathrm{~Hz})$, it could also be applied to any oscillatory noise function present, e.g. respiratory effects $(0.3 \mathrm{~Hz})$, simply by using the frequency of the oscillation to get an initial estimate of the angular frequency $\omega$.

For our purposes the physiological signals discussed in this manuscript are a source of noise. Conversely, the presence of such signals shows the broad potential of nearinfrared techniques for physiological measurement, particularly as an alternative to some of the current invasive methods. 


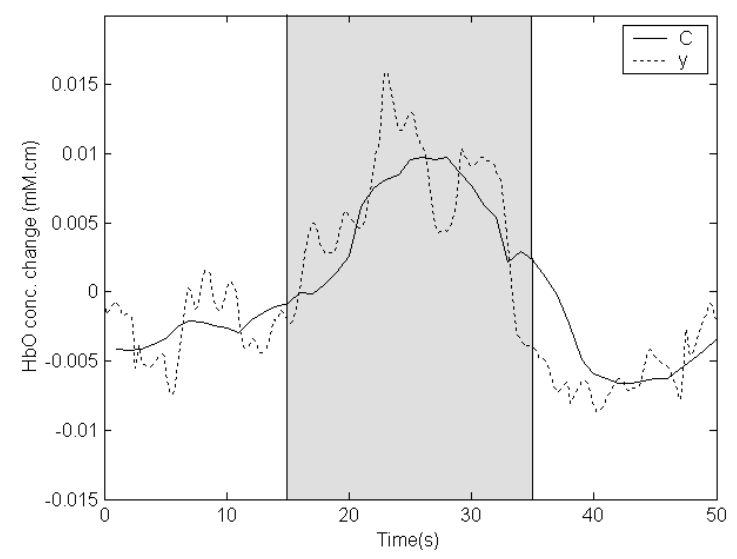

Fig. 5. Oxy-haemoglobin concentration changes before (dashed line), and after signal processing algorithm applied (solid line). Stimulus (hand movement) occurs after $15 \mathrm{~s}$ and lasts for $20 \mathrm{~s}$
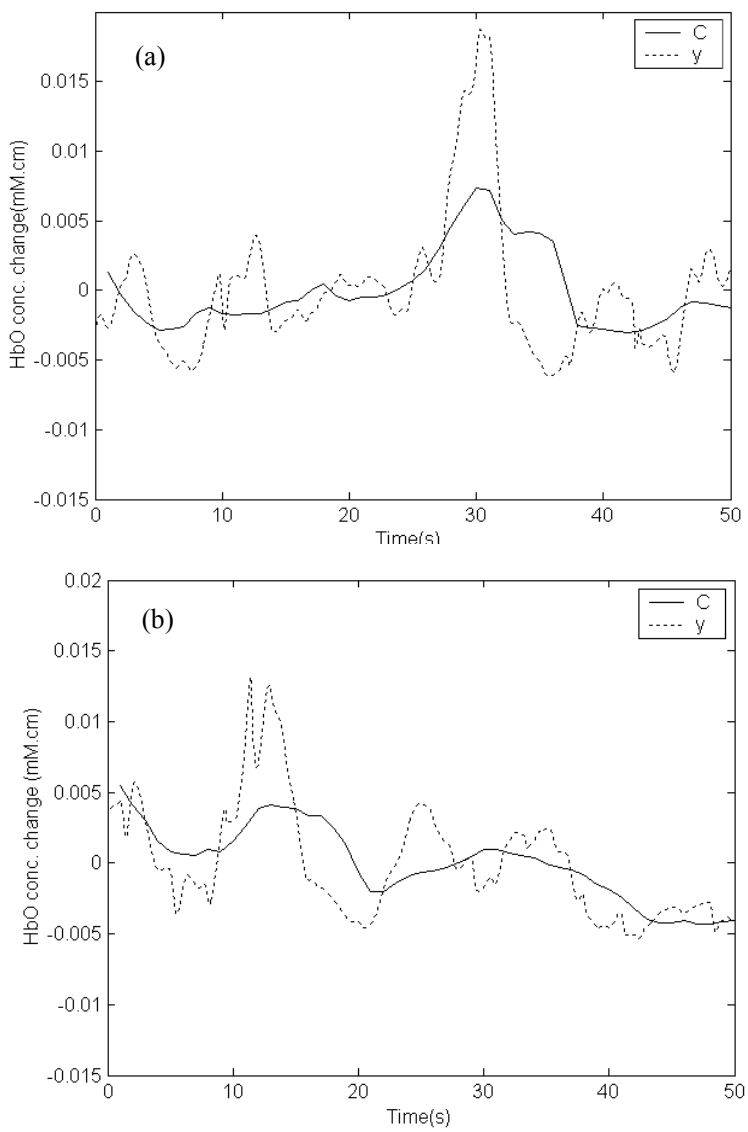

Fig. 6. (a,b) Oxy-haemoglobin concentration changes during rest, before (dashed line), and after signal processing algorithm applied (solid line).

\section{CONCLUSION}

Multiple noise sources, including system noise, motion artifacts and physiological noise, corrupt the detected light signal in near-infrared imaging studies. Efforts can be made to minimize noise within the system, as discussed in Section II, however physiological noise is inherent and can lead to event misclassification in optical BCIs. The noise arises primarily from the cardiac cycle, respiratory functions and cerebral vasomotion. The identification and removal of these endogenous noise sources provides motivation for signal processing algorithms specific to the NIRS domain and more specifically to improve performance of optical BCIs.

\section{ACKNOWLEDGMENT}

This work is funded by the Irish Higher Education Authority.

\section{REFERENCES}

[1] S. Coyle, T. Ward, C. Markham, G. McDarby, "On the Suitability of Near-infrared Systems for Next Generation Brain Computer Interfaces", presented at the World Congress on Medical Physics and Biomedical Engineering, Australia, 2003

[2] C. Roy, C. Sherrington "On the regulation of the blood-supply of the brain", Journal of Physiology vol. 11 pp.85-108, 1890

[3] A. Villringer, J. Planck, C. Hock, L. Schleinkofer, U. Dirnagl, “ Near infrared spectroscopy (NIRS): a new tool to study hemodynamic changes during activation of brain function in human adults", Neuroscience Letters vol. 154, pp.101-104, 1993

[4] S. Coyle, T. Ward, C. Markham, G. McDarby, "Cerebral Blood Flow Changes related to Motor Imagery, using Near-infrared Spectroscopy", presented at the World Congress on Medical Physics and Biomedical Engineering, Sydney, Australia, 2003

[5] G. Gratton, M. Fabiani, T. Elbert, B. Rockstroh, "Seeing right through you: Applications of optical imaging to the study of the human brain." Psychophysiology vol 40, issue 4, pp.487-491, 2003.

[6] C.E. Elwell, R. Springett, E. Hillman, D.T. Delpy, "Oscillations in Cerebral Haemodynamics, Implications for Functional Activation Studies", Advances in Experimental Medicine and Biology, vol. 471, pp. 57-65, 1999

[7] J. E.W. Mayhew et al, "Cerebral vasomotion: A $0.1-\mathrm{Hz}$ Oscillation in Reflected Light Imaging of Neural Activity", Neuroimage, vol. 4, pp. 183-193, 1996

[8] G.A. Millikan, "Physical Instruments for the Biologist", Review of Scientific Instruments, vol. 13, pp. 432-444, 1942

[9] G. Gratton, P.M. Corballis, "Removing the heart from the brain: Compensation for the pulse artifact in the photon migration signal.", Psychophysiology vol. 32, pp. 292-299, 1995

[10] S. Coyle, T. Ward, J. Timoney, C. Markham, "Signal Processing Challenges in Non-invasive Optical Brain Computer Interfacing", unpublished

[11] A. Oppenheim and R. Schafer, Discrete Time Signal Processing. Englewood Cliffs, NJ, Prentice Hall, 1989

[12] H. Obrig et al, "Spontaneous Low Frequency Oscillations of Cerebral Hemodynamics and Metabolism in Human Adults", Neuroimage, vol. 12 pp. 623-639, 2000

[13] R.L. Cooley, "Evidence for a Central Origin of the LowFrequency Oscillation in RR-Interval Variability", Circulation, vol. 98, pp. 556-561, 1998

[14] IEEE Standard for digitizing waveform recorders, IEEE Standard 1057, 1994

[15] I. Tachtsidid, C.E. Elwell, T.S. Leung, C. Lee, M. Smith, D.T. Delpy, " Investigation of cerebral haemodynamics by nearinfrared spectroscopy in young healthy volunteers reveals posture-dependent spontaneous oscillations", Physiological Measurement, vol. 25, pp. 437-445, 2004

[16] P. Händel, "Evaluation of a Standardized Sine Wave Fit Algorithm", Proceedings of the IEEE Nordic Signal Processing Symposium, Sweden, 2000 